\title{
Collective Effects in Casimir-Polder Forces
}

\author{
Kanupriya Sinha, ${ }^{1, *}$ B. Prasanna Venkatesh, ${ }^{2, \dagger}$ and Pierre Meystre ${ }^{3, *}$ \\ ${ }^{1}$ US Army Research Laboratory, Adelphi, Maryland 20783, USA; Joint Quantum Institute, University of Maryland, \\ College Park, Maryland 20742, USA; \\ and Max Planck Institute for the Physics of Complex Systems, Dresden 01187, Germany \\ ${ }^{2}$ Institute for Theoretical Physics, University of Innsbruck, A-6020 Innsbruck, Austria, \\ and Institute for Quantum Optics and Quantum Information of the Austrian Academy of Sciences, A-6020 Innsbruck, Austria \\ ${ }^{3}$ Department of Physics and College of Optical Sciences, University of Arizona, Tucson, Arizona 85721, USA
}

(Received 9 March 2018; published 1 November 2018)

\begin{abstract}
We study cooperative phenomena in the fluctuation-induced forces between a surface and a system of neutral two-level quantum emitters prepared in a coherent collective state, showing that the total Casimir-Polder force on the emitters can be modified via their mutual correlations. Particularly, we find that a one-dimensional chain of emitters prepared in a super- or subradiant state experiences an enhanced or suppressed collective vacuum-induced force, respectively. The collective nature of dispersion forces can be understood as resulting from the interference between the different processes contributing to the surfacemodified resonant dipole-dipole interaction. Such cooperative fluctuation forces depend singularly on the surface response at the resonance frequency of the emitters, thus being easily maneuverable. Our results demonstrate the potential of collective phenomena as a new tool to selectively tailor vacuum forces.
\end{abstract}

DOI: 10.1103/PhysRevLett.121.183605

Introduction.-Collections of atoms and solid-state quantum emitters coupled to waveguides and nanophotonic structures offer a promising platform for scalable quantum information processing [1-4]. The applications of such systems range from building long-ranged quantum networks $[5,6]$, quantum memory devices [7-9], and metrology [10], to facilitating new experimental regimes with exotic lightmatter interactions [11-13]. When interfacing small quantum systems and surfaces at nanoscales, fluctuation-induced phenomena such as vacuum forces [14], surface-modified dissipation [15,16], and decoherence [17] become important considerations. The need to achieve the control and coherence of photonic systems at that scale requires a detailed understanding of these phenomena, so as to determine the extent to which they can be tailored and controlled. In this work, we consider the possibility of using cooperative effects [18-20], as arising from having atoms or emitters in correlated quantum states, to modify fluctuation-induced forces, or Casimir-Polder $(C P)$ forces [21,22].

The study of cooperative effects has a long history in the context of spontaneous emission from a collection of atoms in optical cavities and free space [23-31], and more recently near waveguides $[7,32,33]$. Considering that surface-modified spontaneous emission is the dissipative counterpart to the dispersive vacuum forces [34], one can expect to observe collective effects in dispersion forces as well. When considering vacuum forces, however, the role of quantum coherence within or between the interacting bodies is seldom discussed. While there have been some investigations into the effect of correlations on the van der Waals forces between two atoms [35,36], the effect of spatial wave function coherence on $C P$ forces [37-39], the effect of surface modes on dipole-dipole correlations [40-44], and interference effects in vacuum forces in a three-level system [45], a general analysis of fluctuation-induced forces between an $\mathrm{N}$-particle system prepared in a coherent collective state and a macroscopic body is yet to be explored in detail. The goal of this Letter is to analyze a proof of concept that illustrates cooperative effects in Casimir-Polder forces between a surface and a system of $N$ two-level quantum emitters prepared in a Dicke state [23].

Model.-We consider a one-dimensional chain of $N$ twolevel quantum emitters with ground and excited levels $|g\rangle_{n}$ and $|e\rangle_{n}$ at a distance $z_{0}$ from the surface of a planar halfspace medium, with the emitters separated by a distance $x_{0}$ from one another [see Fig. 1(a)]. We assume that the halfspace $z<0$ is occupied by a medium of dielectric permittivity $\epsilon(\omega)$, while the upper half-space is vacuum. The levels $|g\rangle_{n}$ and $|e\rangle_{n}$ are coupled via an electric-dipole transition with resonance transition frequency $\omega_{0}$ and spontaneous emission rate $\Gamma_{0}$, with $\hat{\sigma}_{n}^{+}=\left(\hat{\sigma}_{n}^{-}\right)^{\dagger}=|e\rangle_{n}\left\langle\left. g\right|_{n}\right.$ being the ladder operators for the corresponding transition. Defining the collective spin operators $\hat{J}_{k} \equiv \sum_{n=1}^{N} \hat{\sigma}_{n}^{k}(k \in\{x, y, z\})$, the Dicke states $|J, M\rangle$ correspond to [23]

$$
\begin{aligned}
& \hat{\mathbf{J}}^{2}|J, M\rangle=J(J+1)|J, M\rangle \quad \text { and } \\
& \hat{J}_{z}|J, M\rangle=M|J, M\rangle .
\end{aligned}
$$

The total Hamiltonian for the system of emitters and the electromagnetic (EM) field is $\hat{H}=\hat{H}_{S}+\hat{H}_{F}+\hat{H}_{\text {int }}$, where 


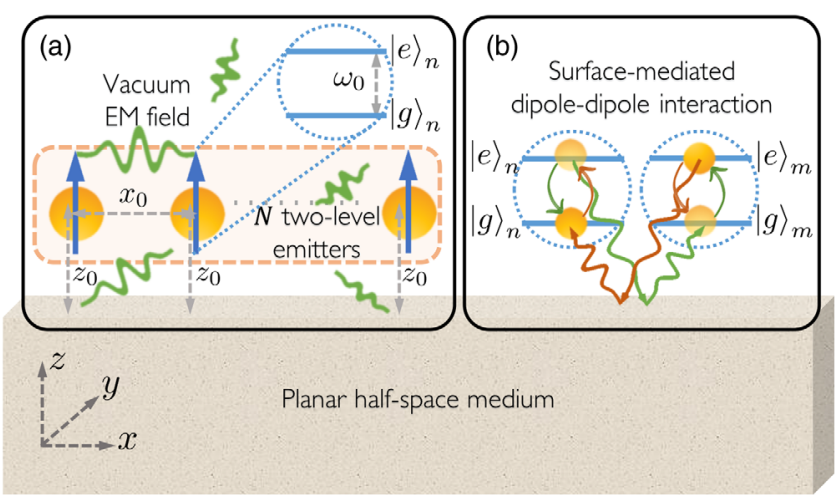

FIG. 1. (a) Schematic representation of $N$ two-level quantum emitters prepared in a collective state, interacting with the vacuum EM field in the presence of a planar half-space medium. (b) Constructive (destructive) interference between the two processes shown in green and red leads to superradiance (subradiance) in the surface-mediated resonant dipole-dipole interactions.

$\hat{H}_{S}=\sum_{n=1}^{N} \hbar \omega_{0} \hat{\sigma}_{n}^{+} \hat{\sigma}_{n}^{-}$is the Hamiltonian for the two-level emitters and $\hat{H}_{F}$ is the Hamiltonian for the medium-assisted EM field, which we assume to be in the vacuum state. The electric-dipole interaction Hamiltonian between the emitters and the EM field is $\hat{H}_{\text {int }}=-\sum_{n=1}^{N} \hat{\mathbf{d}}_{n} \cdot \hat{\mathbf{E}}\left(\mathbf{r}_{n}\right)$, where $\hat{\mathbf{d}}_{n}=\mathbf{d}_{n} \hat{\sigma}_{n}^{+}+\mathbf{d}_{n}^{*} \hat{\sigma}_{n}^{-}$is the electric-dipole operator for the $n$th emitter and $\hat{\mathbf{E}}\left(\mathbf{r}_{n}\right)$ is the electric field at the position $\mathbf{r}_{n}$ of the $n$th emitter in the presence of the surface (see Ref. [46] for further details). We assume the dipole moments of all the emitters $\mathbf{d}_{n} \equiv d_{0} \mathbf{e}_{z}$ to be equal in magnitude and aligned along the $z$ direction [46].

The dynamics of the density matrix $\hat{\rho}_{S}$ of the emitters, after tracing out the EM field, is described by the BornMarkov master equation [48],

$$
\frac{d \hat{\rho}_{S}}{d t}=-\frac{i}{\hbar}\left[\hat{H}_{S}^{\prime}, \hat{\rho}_{S}\right]+\mathcal{L}_{S}^{\prime}\left[\hat{\rho}_{S}\right]
$$

where $\hat{H}_{S}^{\prime}$ is the effective Hamiltonian for the emitters in the interaction picture:

$\hat{H}_{S}^{\prime}=\hbar\left(\sum_{n=1}^{N} \Omega_{n}^{(+)} \hat{\sigma}_{n}^{+} \hat{\sigma}_{n}^{-}+\Omega_{n}^{(-)} \hat{\sigma}_{n}^{-} \hat{\sigma}_{n}^{+}+\sum_{m>n} \Omega_{m n} \hat{\sigma}_{m}^{-} \hat{\sigma}_{n}^{+}\right)$.

Here, $\Omega_{n}^{(-)}=\left[\mu_{0} \omega_{0} /(\hbar \pi)\right] \int_{0}^{\infty} d \xi\left[\xi^{2} /\left(\xi^{2}+\omega_{0}^{2}\right)\right] \mathbf{d}_{n}^{*} \cdot \overline{\bar{G}}_{\mathrm{sc}}\left(\mathbf{r}_{n}\right.$, $\left.\mathbf{r}_{n}, i \xi\right) \cdot \mathbf{d}_{n}$ and $\Omega_{n}^{(+)}=-\Omega_{n}^{(-)}+\Omega_{n}^{(\text {res })}$ are the CasimirPolder shifts for the ground and excited states of the $n$th emitter, respectively. These shifts correspond to processes wherein the $n$th dipole emits and reabsorbs a photon that is scattered off the surface, with the photon propagator given by the scattering Green's tensor $\overline{\bar{G}}_{\mathrm{sc}}\left(\mathbf{r}, \mathbf{r}^{\prime}, \omega\right)$ defined as the solution to the homogeneous Helmholtz equation [49,50]: $\boldsymbol{\nabla} \times \boldsymbol{\nabla} \times \overline{\bar{G}}_{\mathrm{sc}}\left(\mathbf{r}, \mathbf{r}^{\prime}, \omega\right)-\epsilon(\mathbf{r}, \omega) \frac{\omega^{2}}{c^{2}} \overline{\bar{G}}_{\mathrm{sc}}\left(\mathbf{r}, \mathbf{r}^{\prime}, \omega\right)=0$.

Here, $\epsilon(\mathbf{r}, \omega)$ is the space-dependent permittivity of the medium. Note that in addition to the broadband offresonant contribution $\Omega_{n}^{(-)}$, the excited state has a resonant contribution [51],

$$
\Omega_{n}^{(\mathrm{res})} \equiv-\frac{\mu_{0} \omega_{0}^{2}}{\hbar} \operatorname{Re}\left[\mathbf{d}_{n}^{*} \cdot \overline{\bar{G}}_{\mathrm{sc}}\left(\mathbf{r}_{n}, \mathbf{r}_{n}, \omega_{0}\right) \cdot \mathbf{d}_{n}\right],
$$

that depends on the response of the environment at the transition frequency $\omega_{0}$ of the emitters.

The surface-modified resonant dipole-dipole interaction frequency $\Omega_{m n}$ between the emitters $n$ and $m$ can be expressed [52,53] as the sum of a contribution $\Omega_{m n}^{(\text {free })}$ from the resonant exchange of excitation between the two dipoles via a photon propagating in free space, and a contribution $\Omega_{m n}^{(\mathrm{sc})}$ from a photon scattered off the surface, see Fig. 1(b), with

$\Omega_{m n}^{\text {(sc,free) }}=-\frac{\mu_{0} \omega_{0}^{2}}{\hbar} \operatorname{Re}\left[\mathbf{d}_{m}^{*} \cdot \overline{\bar{G}}_{\mathrm{sc}, \text { free }}\left(\mathbf{r}_{m}, \mathbf{r}_{n}, \omega_{0}\right) \cdot \mathbf{d}_{n}\right]$.

Finally, the surface-modified Liouvillian is given by

$\mathcal{L}_{S}^{\prime}\left[\rho_{S}\right]=\sum_{m, n} \frac{\Gamma_{m n}}{2}\left(2 \hat{\sigma}_{m}^{-} \rho_{S} \hat{\sigma}_{n}^{+}-\hat{\sigma}_{m}^{+} \hat{\sigma}_{n}^{-} \rho_{S}-\rho_{S} \hat{\sigma}_{m}^{+} \hat{\sigma}_{n}^{-}\right)$,

where $\Gamma_{n n}$ is the spontaneous emission rate for the excited state of the $n$th emitter, and $\Gamma_{m n}=\Gamma_{m n}^{(\mathrm{free})}+\Gamma_{m n}^{(\mathrm{sc})}$ is the dissipative coupling coefficient between emitters $n$ and $m$, with

$$
\Gamma_{m n}^{(\text {sc,free })}=\frac{2 \mu_{0} \omega_{0}^{2}}{\hbar} \operatorname{Im}\left[\mathbf{d}_{m}^{*} \cdot \overline{\bar{G}}_{\mathrm{sc}, \text { free }}\left(\mathbf{r}_{m}, \mathbf{r}_{n}, \omega_{0}\right) \cdot \mathbf{d}_{n}\right] .
$$

From Eqs. (5) and (8) we see that the dissipative coefficients $\Gamma_{n n}^{(\mathrm{sc})}$ and $\Gamma_{m n}^{(\mathrm{sc}, \text { free })}$ are related to the resonant dispersive shift $\Omega_{n}^{(\text {res })}$ and the dipole-dipole interactions $\Omega_{m n}^{\text {(sc,free) }}$, respectively, being the real and imaginary parts of the same response function [58,59]. As we show below, this implies that a collective enhancement or suppression of resonant van der Waals forces is concomitant with the cooperative behavior of spontaneous emission.

Results.-We define the total $C P$ force for the system of emitters in a state $\hat{\rho}_{S}$ as $F_{\mathrm{CP}}\left[\hat{\rho}_{S}\right]=-(\partial / \partial z) \operatorname{Tr}\left[\hat{H}_{S}^{\prime} \hat{\rho}_{S}\right]$, so that

$$
\begin{aligned}
F_{\mathrm{CP}}\left[\hat{\rho}_{S}\right]= & -\hbar \sum_{n=1}^{N}\left[\frac{\partial}{\partial z} \Omega_{n}^{(+)}\left\langle\hat{\sigma}_{n}^{+} \hat{\sigma}_{n}^{-}\right\rangle+\frac{\partial}{\partial z} \Omega_{n}^{(-)}\left\langle\hat{\sigma}_{n}^{-} \hat{\sigma}_{n}^{+}\right\rangle\right] \\
& -\hbar \sum_{m>n} \frac{\partial}{\partial z} \Omega_{m n}^{(\mathrm{sc})}\left\langle\hat{\sigma}_{m}^{-} \hat{\sigma}_{n}^{+}+\hat{\sigma}_{n}^{-} \hat{\sigma}_{m}^{+}\right\rangle
\end{aligned}
$$


where all the averages are taken over the density operator $\hat{\rho}_{S}$. The first term corresponds to the $C P$ forces on the individual emitters and the second term to the contribution from surface-modified dipole-dipole interactions.

Focusing on that term we observe that while the operator average $\left(\left\langle\hat{\sigma}_{m}^{-} \hat{\sigma}_{n}^{+}+\hat{\sigma}_{n}^{-} \hat{\sigma}_{m}^{+}\right\rangle\right)$depends on the correlations between the dipoles in the state $\hat{\rho}_{S}$, the surface-modified dipole-dipole frequency $\Omega_{m n}^{(\mathrm{sc})}$ depends on the distance of the emitters from the surface. Hence, by preparing the emitters in a suitable collective state $\hat{\rho}_{S}$, the $C P$ force on an ensemble can be modified. Since this modification depends only on the resonant frequency response of the surface, as evident from Eq. (6), it can thus be tailored easily by engineering surface resonances around the resonance frequency of the emitters. This is one of the main results of this Letter.

As a first illustration consider two emitters prepared near a metal surface in one of the four internal states $\left|\Psi_{g}\right\rangle \equiv|g g\rangle$, $\left|\Psi_{e}\right\rangle \equiv|e e\rangle,\left|\Psi_{\text {sup }}\right\rangle \equiv(|e g\rangle+|g e\rangle) / \sqrt{2}$, or $\left|\Psi_{\text {sub }}\right\rangle \equiv(|e g\rangle-$ $|g e\rangle) / \sqrt{2}$. We assume the surface to be described by the Drude model with permittivity $\epsilon(\omega)=1-\omega_{p}^{2} /\left(\omega^{2}+i \omega \gamma\right)$, where $\omega_{p}$ and $\gamma$ are the plasma frequency and loss parameter for the metal, respectively. From Eq. (9) it follows that the force $F_{g(e)}$ for the state $\left|\Psi_{g(e)}\right\rangle$ is the sum of the forces on the individual emitters in the ground (excited) state:

$F_{e g}=-\hbar \frac{\partial}{\partial z}\left[\Omega_{1}^{( \pm)}+\Omega_{2}^{( \pm)}\right] \approx-\frac{9 \omega_{p} \hbar \Gamma_{0} k_{0}}{32\left(\omega_{p} \mp \sqrt{2} \omega_{0}\right) \tilde{z}_{0}^{4}}$.

Here the approximate second expression corresponds to the nonretarded, or near-field, limit of the $C P$ force valid in the emitters-surface distance regime $\tilde{z}_{0} \equiv k_{0} z_{0} \ll 1$, with $k_{0} \equiv \omega_{0} / c \quad[22,46,49]$.

In contrast, the force on the super- and subradiant states,

$$
F_{\text {sup sub }}=-\frac{\hbar}{2} \frac{\partial}{\partial z}\left[\Omega_{1}^{(\text {res })}+\Omega_{2}^{(\text {res })} \pm 2 \Omega_{12}^{(\text {sc })}\right],
$$

includes a contribution that depends on the surfacemediated dipole-dipole interaction in addition to the resonant $C P$ shifts of the individual emitters. In the nonretarded limit, it can be written as

$$
\left.F_{\text {sup }}^{\text {sub }} \underset{\text { क }}{ } \approx 1 \pm f\left(\tilde{x}_{0}, \tilde{z}_{0}\right)\right],
$$

where we have introduced the asymptotic force for infinitely separated emitters,

$$
F_{\infty} \equiv-\frac{9 \omega_{p}^{2} \hbar \Gamma_{0} k_{0}}{16\left(\omega_{p}^{2}-2 \omega_{0}^{2}\right) \tilde{z}_{0}^{4}},
$$

and

$$
f\left(\tilde{x}_{0}, \tilde{z}_{0}\right) \equiv \frac{8 \tilde{z}_{0}^{4}}{3} \int_{0}^{\infty} d \kappa \kappa e^{-2 \kappa \tilde{z}_{0}}\left(\kappa^{2}+1\right) J_{0}\left(\tilde{x}_{0} \sqrt{\kappa^{2}+1}\right)
$$

quantifies the cooperativity due to geometric configuration of the dipoles, with $\tilde{x}_{0} \equiv k_{0} x_{0}$. For coincident dipoles and to lowest order in $\tilde{z}_{0}, \lim _{x_{0} \rightarrow 0} f\left(\tilde{x}_{0}, \tilde{z}_{0}\right) \approx 1$.

As illustrated in Fig. 2(a), at small emitter separations $\left(x_{0} \lesssim z_{0}\right)$ the cooperative contribution leads to an enhanced and suppressed $C P$ force for the super- and subradiant state, respectively. For larger separations, $\lim _{x_{0} \rightarrow \infty} f\left(\tilde{x}_{0}, \tilde{z}_{0}\right) \approx 0$ and the interference effect in the resonant dipole-dipole interaction is attenuated, such that the super- and subradiant states experience an incoherent average of the ground and excited state forces, i.e., $F_{\text {sup,sub }} \approx\left(F_{g}+F_{e}\right) / 2=F_{\infty}$. This is generally true for a state $\left|\Psi_{\theta, \phi}\right\rangle \equiv \cos \theta|e g\rangle+e^{i \phi} \sin \theta|g e\rangle$ with a single shared excitation between the emitters. We note that the total force
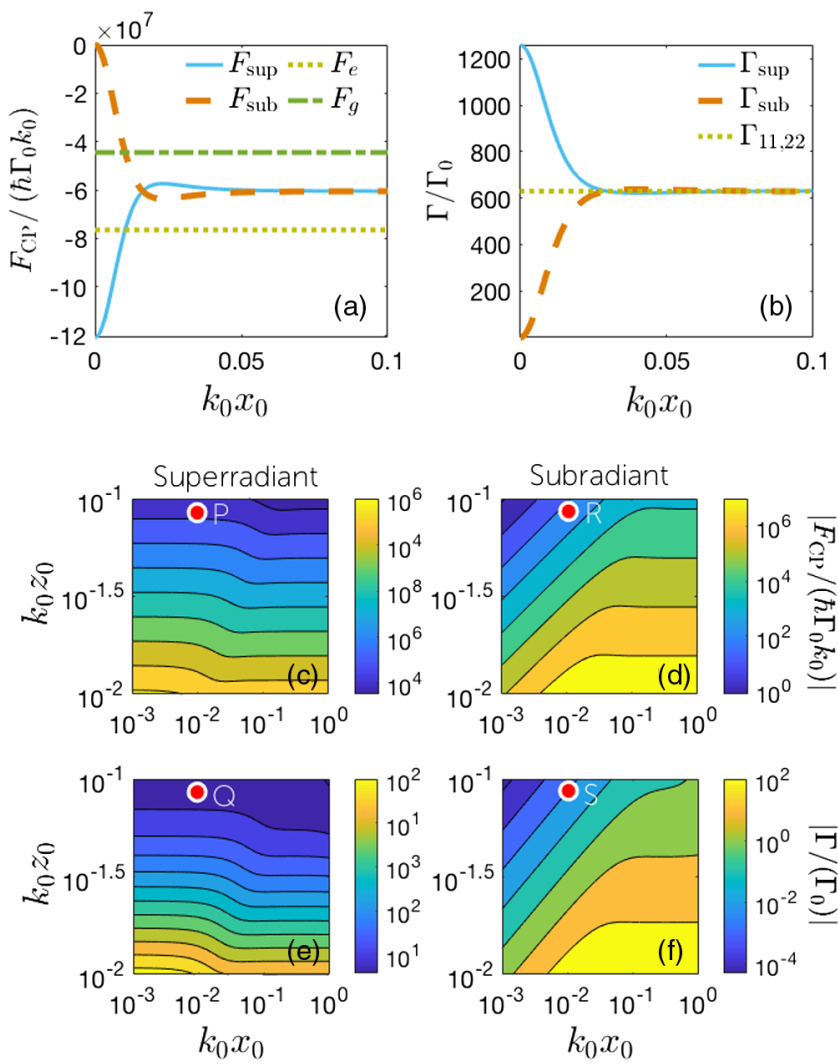

FIG. 2. (a) Collective Casimir-Polder force (in units of $\hbar \Gamma_{0} k_{0}$ ) and (b) spontaneous emission (in units of $\Gamma_{0}$ ), on a system of two emitters near a gold surface, as a function of the separation between the emitters. Here the distance of the emitters from the surface is assumed to be $k_{0} z_{0}=0.01$. (c) [(d)] Collective Casimir-Polder force and (e) [(f)] spontaneous emission on two emitters as a function of their distance from the surface and their mutual separation, for the dipoles prepared in the superradiant [subradiant] state $\left|\Psi_{\text {sup }}\right\rangle\left[\left|\Psi_{\text {sub }}\right\rangle\right]$. For reference, the ground state Casimir-Polder force on the two emitters at point $P$ is roughly $\left|F_{g}\right| \sim 10^{4} \hbar \Gamma_{0} k_{0}$. The surface is described by the Drude model with a plasma frequency $\omega_{p} \approx 1.37 \times 10^{16} \mathrm{~Hz}$ $(9 \mathrm{eV})$ and loss parameter $\gamma \approx 5.31 \times 10^{13} \mathrm{~Hz}(35 \mathrm{meV})$ for gold [60]. 
on the state $\left|\Psi_{\theta, \phi}\right\rangle$ is given by $F_{\theta, \phi}=-\hbar(\partial / \partial z)\left[\Omega_{1,2}^{(\mathrm{res})}+\right.$ $\left.\Omega_{12}^{(\text {sc) }} \sin (2 \theta) \cos \phi\right]$, which can vary between the super- and subradiant values in Eq. (12), depending on the relative amplitudes $(\tan \theta)$ and phase $(\cos \phi)$ between the states $|e g\rangle$ and $|g e\rangle$. The collective spontaneous emission for the superradiant (subradiant) state, given by $\Gamma_{\text {sup }}=1 / 2\left[\Gamma_{11}+\right.$ $\left.\Gamma_{22}+2 \Gamma_{12}\right]\left(\Gamma_{\text {sub }}=1 / 2\left[\Gamma_{11}+\Gamma_{22}-2 \Gamma_{12}\right]\right)$ is depicted in Fig. 2(b) [46].

Figures 2(c)-2(f) give a more comprehensive picture of the collective $C P$ forces and spontaneous emission as a function of the geometrical configuration of the dipoles. Assuming the emitter resonant wavelength to be $\lambda_{0} \equiv 2 \pi \mathrm{c} /$ $\omega_{0} \sim 700 \mathrm{~nm}$, we see from the points $R$ and $S$ in Figs. 2(d) and 2(f), respectively, that a subradiant state of two emitters separated by $x_{0} \sim 1 \mathrm{~nm}$, and at a distance $z_{0} \sim 10 \mathrm{~nm}$ from a gold surface, experiences a total force that is suppressed by a factor of $F_{\text {sub }} / F_{g} \sim 10^{-2}$ relative to the ground state van der Waals force, with a spontaneous emission $\Gamma_{\text {sub }} / \Gamma_{0} \sim 10^{-2}$. Hence, subradiant $C P$ forces provide a potential way to avoid both dissipation and undesirable $C P$ attraction.

For a system of $N$ dipoles the $C P$ force on the Dicke superradiant state $|J=N / 2, M=0\rangle$ can be written as

$$
F_{\text {sup }}=-\frac{\hbar}{2} \sum_{n=1}^{N} \frac{\partial \Omega_{n}^{(\mathrm{res})}}{\partial z}-2 \hbar \frac{\left(\begin{array}{c}
N-2 \\
-1+N / 2
\end{array}\right)}{\left(\begin{array}{c}
N / 2 \\
N / 2
\end{array}\right)} \sum_{m>n} \frac{\partial \boldsymbol{\Omega}_{m n}^{(\mathrm{sc})}}{\partial z},
$$

where $\left(\begin{array}{l}N \\ k\end{array}\right)$ is a binomial coefficient. In the limit of superposed dipoles, $x_{0} \rightarrow 0$, it reduces to

$$
\lim _{x_{0} \rightarrow 0} F_{\text {sup }}=-\frac{9 \omega_{p}^{2} \hbar \Gamma_{0} k_{0}}{32\left(\omega_{p}^{2}-2 \omega_{0}^{2}\right) \tilde{z}_{0}^{4}}\left(N+\frac{N^{2}}{2}\right),
$$

which demonstrates the characteristic $N^{2}$ scaling of the collective $C P$ force on the superradiant state, depicted in the inset of Fig. 3, similar to free-space superradiant spontaneous emission at small emitter separations $\left(\tilde{x}_{0} \ll 1\right)$ [24]. We also remark that, for $N>2$, multiple states in the degenerate subspace of subradiant Dicke states with $|J=0, M=0\rangle$ exhibit a suppressed $C P$ force; see Ref. [46].

Discussion.-We have identified collective effects in vacuum-induced dispersion forces that result from the interference between the different channels contributing to the surface-modified resonant dipole-dipole interaction, as sketched in Fig. 1(b). Such cooperative enhancement or suppression of fluctuation forces occurs for the resonant contribution to the total $C P$ force, and can be physically understood as the dispersive counterpart to super- or subradiance in spontaneous emission [see Eq. (12)]. In addition to the quantum correlations [63] in the state of the emitters this contribution to the total $C P$ force depends only on the surface response at the resonance frequency of the emitters; see Eq. (6). It can thus be controlled by suitably

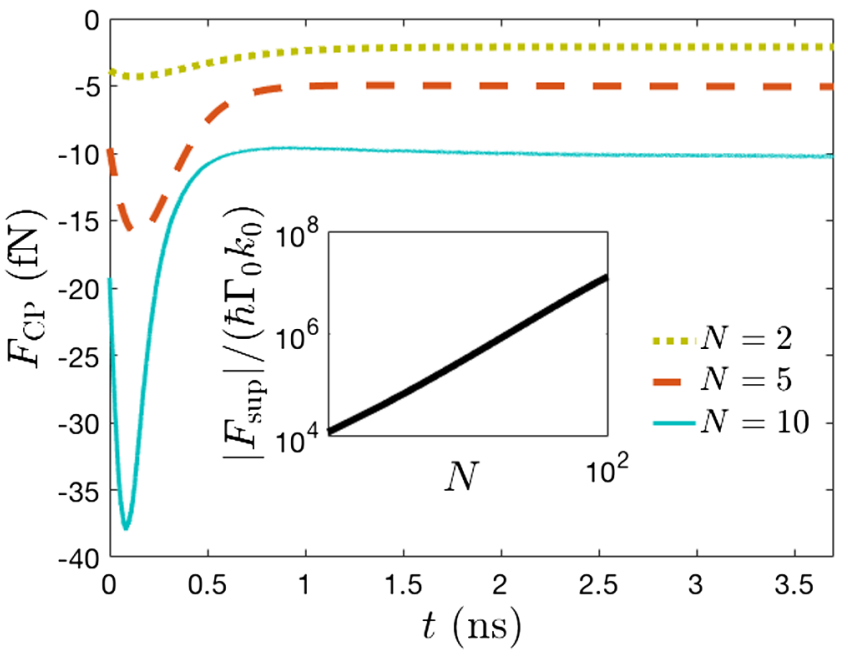

FIG. 3. Superradiant boost of the time-dependent total attractive $C P$ force on a linear chain of $N \mathrm{SiV}$ emitters initially prepared in the excited level of the $737 \mathrm{~nm}$ transition with a lifetime of $1.7 \mathrm{~ns}$ [61], placed $z_{0} \approx 10 \mathrm{~nm}$ from a gold surface [47,62]. The inset shows the absolute value of the maximum boost as a function of the number of emitters, illustrating the $N^{2}$ scaling of the force for the superradiant state $|J=N / 2, M=0\rangle$.

tailoring the response of the surface around the resonant frequency of the emitters.

Given that cooperative effects in optical dipole forces on solid-state emitters in nanodiamonds have been discussed both theoretically and experimentally [67-69], we suggest that it should be possible to observe a boost in the cooperative vacuum-induced forces by placing a similar nanodiamond doped with emitters near a surface. To estimate the feasibility of observing the collectively enhanced $C P$ force, we consider a system of $N$ siliconvacancy $(\mathrm{SiV})$ centers embedded in a cantilever near a metal surface $[70,71]$. We assume that they are initially prepared in the excited state, and solve the superradiance master equation Eq. (2) numerically [72]. As the system decays in a collective manner, it occupies the superradiant manifold transiently and experiences an enhanced $C P$ force, as shown in Fig. 3. For a system of $N=10 \mathrm{SiV}$ centers at a distance of $z_{0} \approx 10 \mathrm{~nm}$ from a gold surface, we find a superradiant boost in the collective $C P$ force of $\Delta F_{\mathrm{CP}} \approx 20 \mathrm{fN}$ over a timescale of $\Delta \tau \approx 0.5 \mathrm{~ns}[73,75]$. The numerical results for the $N=10$ case are from a trajectory simulation averaged over 1000 trajectories, whereas for the smaller $N \leq 6$, a direct simulation of the master equation Eq. (2) was performed. While the magnitude of the enhanced force is large enough to be observable with current technologies [76], the time resolution required to sense the enhancement would appear to pose an experimental challenge.

While we have concentrated here on the specific case of a fully inverted ensemble, a thorough analysis of different initial states will be considered in the future (see Ref. [68] for a pulsed excitation scheme used to observe superradiant emission). We also anticipate that the effects described here 
will be relevant and possibly observable in a variety of other platforms such as van der Waals shifts on atoms placed near optical fibers [32], quantum dot systems [77,78], and superconducting qubits [31].

In terms of potential applications one can speculate that superradiant states could be used to boost hard to observe weak fluctuation forces such as the ones discussed in Ref. [79], as well as a probe of surface properties [80]. More interestingly perhaps, given that subradiant states suppress undesirable Casimir-Polder attraction and exhibit long lifetimes with respect to the single-atom excited states [Fig. 2(f)], they can be a useful resource for trapping particles near surfaces, with potential applications in terms of quantum computation given their robustness to environmental decoherence [81,82].

We are grateful to Ana Asenjo-Garcia, Ania C. Bleszynski Jayich, Mathieu L. Juan, Francesco Piazza, Helmut Ritsch, Oriol Romero-Isart, and Pablo Solano for insightful discussions. B. P. V. was supported by the Austrian Federal Ministry of Science, Research and Economy (BMWFW) and the Austrian Science Fund FWF through projects SFB FoQuS P13. This work was partially supported by the Oak Ridge Associated Universities.

Note added.-The related works in Refs. [83,84] were brought to our attention recently.

*kanu@umd.edu

†Prasanna.Venkatesh@uibk.ac.at

Present address: Indian Institute of Technology, Gandhinagar 382355, India.

*pierre@optics.arizona.edu

[1] K. Nemoto, M. Trupke, S. J. Devitt, A. M. Stephens, B. Scharfenberger, K. Buczak, T. Nöbauer, M. S. Everitt, J. Schmiedmayer, and W. J. Munro, Photonic Architecture for Scalable Quantum Information Processing in Diamond, Phys. Rev. X 4, 031022 (2014).

[2] M. D. Lukin, M. Fleischhauer, R. Cote, L. M. Duan, D. Jaksch, J. I. Cirac, and P. Zoller, Dipole Blockade and Quantum Information Processing in Mesoscopic Atomic Ensembles, Phys. Rev. Lett. 87, 037901 (2001).

[3] N. Y. Yao, L. Jiang, A. V. Gorshkov, P. C. Maurer, G. Giedke, J. I. Cirac, and M. D. Lukin, Scalable architecture for a room temperature solid-state quantum information processor, Nat. Commun. 3, 800 (2012).

[4] K. Hammerer, A. S. Sørensen, and E. S. Polzik, Quantum interface between light and atomic ensembles, Rev. Mod. Phys. 82, 1041 (2010).

[5] H. J. Kimble, The quantum internet, Nature (London) 453, 1023 (2008)

[6] K. Nemoto, M. Trupke, S. J. Devitt, B. Scharfenberger, K. Buczak, J. Schmiedmayer, and W. J. Munro, Photonic quantum networks formed from $\mathrm{NV}^{-}$centers, Sci. Rep. 6, 26284 (2016).

[7] A. Asenjo-Garcia, M. Moreno-Cardoner, A. Albrecht, H. J. Kimble, and D.E. Chang, Exponential Improvement in
Photon Storage Fidelities Using Subradiance and Selective Radiance in Atomic Arrays, Phys. Rev. X 7, 031024 (2017).

[8] S. L. Mouradian, T. Schröder, C. B. Poitras, L. Li, J. Goldstein, E. H. Chen, M. Walsh, J. Cardenas, M. L. Markham, D. J. Twitchen, M. Lipson, and D. Englund, Scalable Integration of Long-Lived Quantum Memories into a Photonic Circuit, Phys. Rev. X 5, 031009 (2015).

[9] M. Steger, K. Saeedi, M. L. W. Thewalt, J. J. L. Morton, H. Riemann, N. V. Abrosimov, P. Becker, and H.-J. Pohl, Quantum information storage for over $180 \mathrm{~s}$ using donor spins in a ${ }^{28} \mathrm{Si}$ "semiconductor vacuum", Science 336, 1280 (2012).

[10] J.-W. Zhou, P.-F. Wang, F.-Z. Shi, P. Huang, X. Kong, X.-K. Xu, Q. Zhang, Z.-X. Wang, X. Rong, and J.-F. Du, Quantum information processing and metrology with color centers in diamonds, Front. Phys. 9, 587 (2014).

[11] J. S. Douglas, H. Habibian, C.-L. Hung, A. V. Gorshkov, H. J. Kimble, and D. E. Chang, Quantum many-body models with cold atoms coupled to photonic crystals, Nat. Photonics 9, 326 (2015).

[12] D. E. Chang, J. I. Cirac, and H. J. Kimble, Self-Organization of Atoms along a Nanophotonic Waveguide, Phys. Rev. Lett. 110, 113606 (2013).

[13] D. E. Chang, J.S. Douglas, A. González-Tudela, C.-L. Hung, and H. J. Kimble, Colloquium: Quantum matter built from nanoscopic lattices of atoms and photons, Rev. Mod. Phys. 90, 031002 (2018).

[14] D. E. Chang, K. Sinha, J. M. Taylor, and H. J. Kimble, Trapping atoms using nanoscale quantum vacuum forces, Nat. Commun. 5, 4343 (2014).

[15] M. S. Yeung and T. K. Gustafson, Spontaneous emission near an absorbing dielectric surface, Phys. Rev. A 54, 5227 (1996).

[16] F. L. Kien, S. D. Gupta, V. I. Balykin, and K. Hakuta, Spontaneous emission of a cesium atom near a nanofiber: Efficient coupling of light to guided modes, Phys. Rev. A 72, 032509 (2005).

[17] R. Fermani, S. Scheel, and P. L. Knight, Trapping cold atoms near carbon nanotubes: Thermal spin flips and Casimir-Polder potential, Phys. Rev. A 75, 062905 (2007).

[18] J. Javanainen and J. Ruostekoski, Light propagation beyond the mean-field theory of standard optics, Opt. Express 24, 993 (2016).

[19] W. Guerin, M. T. Rouabah, and R. Kaiser, Light interacting with atomic ensembles: Collective, cooperative and mesoscopic effects, J. Mod. Opt. 64, 895 (2017).

[20] J. Javanainen, J. Ruostekoski, Y. Li, and S.-M. Yoo, Exact electrodynamics versus standard optics for a slab of cold dense gas, Phys. Rev. A 96, 033835 (2017).

[21] P. W. Milonni, The Quantum Vacuum: An Introduction to Quantum Electrodynamics (Academic, San Diego, 1993).

[22] H. B. G. Casimir and D. Polder, The influence of retardation on the London-van der Waals forces, Phys. Rev. 73, 360 (1948).

[23] R. H. Dicke, Coherence in spontaneous radiation processes, Phys. Rev. 93, 99 (1954).

[24] M. Gross and S. Haroche, Superradiance: An essay on the theory of collective spontaneous emission, Phys. Rep. 93, 301 (1982).

[25] Z. Ficek and R. Tanaś, Entangled states and collective nonclassical effects in two-atom systems, Phys. Rep. 372, 369 (2002). 
[26] R. G. DeVoe and R. G. Brewer, Observation of Superradiant and Subradiant Spontaneous Emission of Two Trapped Ions, Phys. Rev. Lett. 76, 2049 (1996).

[27] D. Bhatti, R. Schneider, S. Oppel, and J. von Zanthier, Directional Dicke Subradiance with Nonclassical and Classical Light Sources, Phys. Rev. Lett. 120, 113603 (2018).

[28] N. Skribanowitz, I. P. Herman, J. C. MacGillivray, and M. S. Feld, Observation of Dicke Superradiance in Optically Pumped HF Gas, Phys. Rev. Lett. 30, 309 (1973).

[29] L. Mandel and E. Wolf, Optical Coherence and Quantum Optics (Cambridge University Press, Cambridge, England, 1995).

[30] A. V. Andreev, V. I. Emel'yanov, and Y. A. Il'nskii, Collective spontaneous emission (Dicke superradiance), Sov. Phys. Usp. 23, 493 (1980).

[31] J. A. Mlynek, A. A. Abdumalikov, C. Eichler, and A. Wallraff, Observation of Dicke superradiance for two artificial atoms in a cavity with high decay rate, Nat. Commun. 5, 5186 (2014).

[32] P. Solano, P. Barberis-Blostein, F. K. Fatemi, L. A. Orozco, and S.L. Rolston, Super-radiance reveals infinite-range dipole interactions through a nanofiber, Nat. Commun. 8, 1857 (2017).

[33] Y. Li and C. Argyropoulos, Controlling collective spontaneous emission with plasmonic waveguides, Opt. Express 24, 26696 (2016).

[34] F. Intravaia, C. Henkel, and M. Antezza, Fluctuationinduced forces between atoms and surfaces: The CasimirPolder interaction, in Casimir Physics, Lecture Notes in Physics, edited by D. Dalvit, P. Milonni, D. Roberts, and F. da Rosa (Springer, Berlin, 2011).

[35] S. Esfandiarpour, R. Bennett, H. Safari, and S. Y. Buhmann, Cavity-QED interactions of two correlated atoms, J. Phys. B 51, 094004 (2018).

[36] R. O. Behunin and B. L. Hu, Nonequilibrium forces between neutral atoms mediated by a quantum field, Phys. Rev. A 82, 022507 (2010).

[37] V. Klimov, V. S. Letokhov, and M. Ducloy, Quasi orbital motion of ultra cold excited atomic dipole near dielectric microsphere, Eur. Phys. J. D 5, 345 (1999).

[38] J. Schiefele and C. Henkel, Bose-Einstein condensate near a surface: Quantum field theory of the Casimir-Polder interaction, Phys. Rev. A 82, 023605 (2010).

[39] J. Schiefele and C. Henkel, Bosonic enhancement of spontaneous emission near an interface, Phys. Lett. A 375, 680 (2011).

[40] V. Klimov, S. K. Sekatskii, and G. Dietler, Coherent fluorescence resonance energy transfer between two dipoles: Full quantum electrodynamics approach, J. Mod. Opt. 51, 1919 (2004).

[41] A. González-Tudela, D. Martín-Cano, E. Moreno, L. MartínMoreno, C. Tejedor, and F. J. García-Vidal, Entanglement of Two Qubits Mediated by One-Dimensional Plasmonic Waveguides, Phys. Rev. Lett. 106, 020501 (2011).

[42] J. Xu, M. Al-Amri, Y. Yang, S.-Y. Zhu, and M. S. Zubairy, Entanglement generation between two atoms via surface modes, Phys. Rev. A 84, 032334 (2011).

[43] M. Otten, R. A. Shah, N. F. Scherer, M. Min, M. Pelton, and S. K. Gray, Entanglement of two, three, or four plasmonically coupled quantum dots, Phys. Rev. B 92, 125432 (2015).

[44] D. Martín-Cano, A. González-Tudela, L. Martín-Moreno, F. J. García-Vidal, C. Tejedor, and E. Moreno, Dissipationdriven generation of two-qubit entanglement mediated by plasmonic waveguides, Phys. Rev. B 84, 235306 (2011).

[45] J. Xu, S. Chang, Y. Yang, and M. Al-amri, Casimir-Polder force on a $V$-type three-level atom near a structure containing left-handed materials, Phys. Rev. A 93, 012514 (2016).

[46] See Supplemental Material at http://link.aps.org/ supplemental/10.1103/PhysRevLett.121.183605 for additional details on the medium-assisted EM field, the derivation of the superradiance master equation near a surface, effect of dipole orientation on the collective $C P$ force, collective spontaneous emission near a metal surface, and the $C P$ force on subradiant Dicke states of $N$ emitters, which includes Refs. [20,25,43-47].

[47] H. Ishiwata, M. Nakajima, K. Tahara, H. Ozawa, T. Iwasaki, and M. Hatano, Perfectly aligned shallow ensemble nitrogen-vacancy centers in (111) diamond, Appl. Phys. Lett. 111, 043103 (2017).

[48] H.-P. Breuer and F. Petruccione, Theory of Open Quantum Systems (Oxford University Press, New York, 2002).

[49] S. Y. Buhmann, Dispersion Forces I (Springer-Verlag, Berlin, 2012).

[50] T. Gruner and D. G. Welsch, Green-function approach to the radiation-field quantization for homogeneous and inhomogeneous Kramers-Kronig dielectrics, Phys. Rev. A 53, 1818 (1996).

[51] S. Y. Buhmann, Dispersion Forces II (Springer-Verlag, Berlin, 2012).

[52] We have ignored the fourth-order van der Waals interactions between the dipoles, which scale as $\Omega_{m n}^{(4)} \sim 1 / x_{0}^{6}$, assuming that those contributions are weaker in comparison to the second-order interactions for the relevant dipole separations considered here. This is observing that $k_{0} x_{0} \gtrsim$ $\left(\Gamma_{0} / \omega_{0}\right)^{1 / 3}$, given that the second- and fourth-order contributions scale roughly as $\Omega_{m n}^{(2)} \sim\left[\hbar \Gamma_{0} /\left(k_{0} x_{0}\right)^{3}\right]$ and $\Omega_{m n}^{(4)} \sim\left\{\hbar \Gamma_{0}^{2} /\left[\omega_{0}\left(k_{0} x_{0}\right)^{6}\right]\right\}[49]$.

[53] We have also ignored here the long-range van der Waals tail contributions as discussed in Refs. [54-56]. Noting that in the distance regime of interest $a_{0} \ll k_{0} x_{0} \ll \hbar c / \mathcal{L}$, where $a_{0}$ is the Bohr radius and $\mathcal{L}$ is the Lamb energy $(\sim 1 \mathrm{GHz}$ [57]), either the van der Waals potential or the Wick-rotated contribution [55] dominates. We see that both these contributions scale roughly as $\sim\left[E_{h}\left(a_{0} k_{0}\right)^{6} /\left(k_{0} x_{0}\right)^{6}\right]$, where $E_{h}=\hbar c \alpha / a_{0}$ refers to the Hartree energy (with $\alpha$ as the fine-structure constant), such that they can be ignored in comparison to the resonant second-order contribution in the pertinent distance regime $k_{0} x_{0} \gtrsim\left\{\left[c \alpha\left(a_{0} k_{0}\right)^{6}\right] / a_{0} \Gamma_{0}\right\}^{1 / 3}$.

[54] M. Donaire, R. Guérout, and A. Lambrecht, Quasiresonant van der Waals Interaction between Nonidentical Atoms, Phys. Rev. Lett. 115, 033201 (2015).

[55] U. D. Jentschura and V. Debierre, Long-range tails in van der Waals interactions of excited-state and ground-state atoms, Phys. Rev. A 95, 042506 (2017).

[56] U. D. Jentschura, C. M. Adhikari, and V. Debierre, Virtual Resonant Emission and Oscillatory Long-Range Tails in van der Waals Interactions of Excited States: QED 
Treatment and Applications, Phys. Rev. Lett. 118, 123001 (2017).

[57] C. M. Adhikari, V. Debierre, A. Matveev, N. Kolachevsky, and U. D. Jentschura, Long-range interactions of hydrogen atoms in excited states. I. $2 S-1 S$ interactions and Dirac- $\delta$ perturbations, Phys. Rev. A 95, 022703 (2017).

[58] H. T. Dung, L. Knöll, and D.-G. Welsch, Resonant energy exchange between atoms in dispersing and absorbing surroundings, Opt. Spectrosc. 94, 829 (2003).

[59] H. T. Dung, L. Knöll, and D.-G. Welsch, Resonant dipoledipole interaction in the presence of dispersing and absorbing surroundings, Phys. Rev. A 66, 063810 (2002).

[60] I. Pirozhenko, A. Lambrecht, and V. B. Svetovoy, Sample dependence of the Casimir force, New J. Phys. 8, 238 (2006).

[61] Y. Zhou, A. Rasmita, K. Li, Q. Xiong, I. Aharonovich, and W.-B. Gao, Coherent control of a strongly driven silicon vacancy optical transition in diamond, Nat. Commun. 8, 14451 (2017)

[62] S. Choi, J. Choi, R. Landig, G. Kucsko, H. Zhou, J. Isoya, F. Jelezko, S. Onoda, H. Sumiya, V. Khemani, C. von Keyserlingk, N. Y. Yao, E. Demler, and M. D. Lukin, Observation of discrete time-crystalline order in a disordered dipolar many-body system, Nature (London) 543, 221 (2017).

[63] We remark that the resonant contribution to the total $C P$ shift corresponds to the classical analog of a dipole interaction energy near a surface [64]; thus there should exist a similar cooperative dispersion force for a collection of classical dipoles with phase coherence radiating near a surface $[65,66]$.

[64] E. A. Hinds and V. Sandoghdar, Cavity QED level shifts of simple atoms, Phys. Rev. A 43, 398 (1991).

[65] D. J. W. Dikken, J. P. Korterik, F. B. Segerink, J. L. Herek, and J.C. Prangsma, A phased antenna array for surface plasmons, Sci. Rep. 6, 25037 (2016).

[66] V. N. Pustovit and T. V. Shahbazyan, Cooperative Emission of Light by an Ensemble of Dipoles Near a Metal Nanoparticle: The Plasmonic Dicke Effect, Phys. Rev. Lett. 102, 077401 (2009).

[67] M. L. Juan, C. Bradac, B. Besga, M. Johnsson, G. Brennen, G. Molina-Terriza, and T. Volz, Cooperatively enhanced dipole forces from artificial atoms in trapped nanodiamonds, Nat. Phys. 13, 241 (2017).

[68] C. Bradac, M. T. Johnsson, M. van Breugel, B. Q. Baragiola, R. Martin, M. L. Juan, G. K. Brennen, and T. Volz, Roomtemperature spontaneous superradiance from single diamond nanocrystals, Nat. Commun. 8, 1205 (2017).

[69] B. P. Venkatesh, M. L. Juan, and O. Romero-Isart, Cooperative Effects in Closely Packed Quantum Emitters with Collective Dephasing, Phys. Rev. Lett. 120, 033602 (2018).

[70] M. Pelliccione, A. Jenkins, P. Ovartchaiyapong, C. Reetz, E. Emmanouilidou, N. Ni, and A. C. B. Jayich, Scanned probe imaging of nanoscale magnetism at cryogenic temperatures with a single-spin quantum sensor, Nat. Nanotechnol. 11, 700 (2016).

[71] J. Kleinlein, T. Borzenko, F. Münzhuber, J. Brehm, T. Kiessling, and L. Molenkamp, NV-center diamond cantilevers: Extending the range of available fabrication methods, Microelectron. Eng. 159, 70 (2016).

[72] J. R. Johansson, P. D. Nation, and F. Nori, QuTiP 2: A Python framework for the dynamics of open quantum systems, Comput. Phys. Commun. 184, 1234 (2013).

[73] For context, the $C P$ force on a single emitter in the ground at a distance of $z_{0} \approx 10 \mathrm{~nm}$ is $F_{\mathrm{CP}} \approx 2 \mathrm{fN}$. The $C P$ force on a typical nanodiamond of diameter $\approx 30 \mathrm{~nm}[74]$ at a distance of $z_{0} \approx 10 \mathrm{~nm}$ from a gold surface is $\left|F_{\mathrm{CP} \text {,bulk }}\right| \sim 1 \mathrm{nN}$.

[74] M. L. Juan, G. Molina-Terriza, T. Volz, and O. RomeroIsart, Near-field levitated quantum optomechanics with nanodiamonds, Phys. Rev. A 94, 023841 (2016).

[75] We have ignored here the force on the emitters due to photon scattering and recoil. This can be estimated from considering the points $\mathrm{P}$ and $Q$ in Figs. 2(c) and 2(e). We note that the collective rate of photon emission at point $Q$ is enhanced to $\Gamma \sim 10 \Gamma_{0}$. This would roughly correspond to a recoil force magnitude of $\left|F_{\text {rec }}\right| \sim \hbar \Gamma k_{\text {rec }}$, where $k_{\text {rec }} \approx \sqrt{3} / z_{0}$ is the effective momentum associated with a scattered photon near a surface (see the Supplemental Material in Ref. [14], for example). The recoil force around point $Q$ can then be estimated as $\left|F_{\text {rec }}\right| \sim\left[10 /\left(k_{0} z_{0}\right)\right] \hbar \Gamma_{0} k_{0} \sim 20 \hbar \Gamma_{0} k_{0}$, which is significantly smaller than the dispersive superradiant force of $\left|F_{\text {sup }}\right| \sim 10^{5} \hbar \Gamma_{0} k_{0}$, as can be seen from point $P$ of Fig. 2(a).

[76] M. S. J. Barson, P. Peddibhotla, P. Ovartchaiyapong, K. Ganesan, R. L. Taylor, M. Gebert, Z. Mielens, B. Koslowski, D. A. Simpson, L. P. McGuinness, J. McCallum, S. Prawer, S. Onoda, T. Ohshima, A. C. B. Jayich, F. Jelezko, N. B. Manson, and M. W. Doherty, Nanomechanical sensing using spins in diamond, Nano Lett. 17, 1496 (2017).

[77] M. Scheibner, T. Schmidt, L. Worschech, A. Forchel, G. Bacher, T. Passow, and D. Hommel, Superradiance of quantum dots, Nat. Phys. 3, 106 (2007).

[78] M. Praveena, T. Phanindra Sai, R. Dutta, A. Ghosh, and J. K. Basu, Electrically tunable enhanced photoluminescence of semiconductor quantum dots on graphene, ACS Photonics 4, 1967 (2017).

[79] K. Sinha, Repulsive vacuum-induced forces on a magnetic particle, Phys. Rev. A 97, 032513 (2018).

[80] A. Ariyaratne, D. Bluvstein, B. A. Myers, and A. C. B. Jayich, Nanoscale electrical conductivity imaging using a nitrogen-vacancy center in diamond, Nat. Commun. 9, 2406 (2018).

[81] S. Krämer, L. Ostermann, and H. Ritsch, Optimized geometries for future generation optical lattice clocks, Europhys. Lett. 114, 14003 (2016).

[82] D. A. Lidar and K. Birgitta Whaley, in Irreversible Quantum Dynamics, Springer Lecture Notes in Physics, edited by F. Benatti and R. Floreanini (Springer, Berlin, 2003), Vol. 622.

[83] S. Fuchs and S. Y. Buhmann, Purcell-Dicke effect for an atomic ensemble near a surface, arXiv:1804.01265.

[84] R. Jones, J. A. Needham, I. Lesanovsky, F. Intravaia, and B. Olmos, Modified dipole-dipole interaction and dissipation in an atomic ensemble near surfaces, Phys. Rev. A 97, 053841 (2018). 